\title{
CÁLCULO DE LAS BETAS DEL CAPITAL ASSET PRICING MODEL COMO INDICADOR DE RENTABILIDAD DE LAS EMPRESAS VINCULADAS A LA BOLSA DE VALORES DE ECUADOR
}

\author{
Valverde Jenny ${ }^{1}$ y Caicedo Francisco ${ }^{2}$. \\ $\left\{\right.$ jmvalverde $3^{1}$, fmcaicedo 2$\}$ @espe.edu.ec \\ https://orcid.org/0000-0002-1065-7896 ${ }^{1}$, https://orcid.org/0000-0002-4860-6787 ${ }^{2}$ \\ Universidad de las Fuerzas Armadas ESPE \\ Sangolquí - Ecuador
}

Recibido (09/10/20), Aceptado (28/11/20)

\begin{abstract}
Resumen: El riesgo al invertir en títulos-valeres pertenecientes al mercado de capitales no solo depende de los resultados de los emisores, sino, del entorno económico. Bajo este contexto, el objetivo del estudio fue calcular las betas mediante la aplicación del Capital Asset Princing Model (CAPM), para conocer la influencia rentable de las empresas vinculadas a la Bolsa de Valores de Ecuador. La muestra fue de 35 empresas tenedoras de acciones durante el periodo 2014-2019. La investigación se desarrolló bajo un enfoque cuantitativo, mediante un alcance correlacional entre las variables riesgo sistemático y la rentabilidad. Los resultados evidenciaron que utilizar el riesgo sistemático no es considerado como un modelo de valuación de activos financieros fuertes, debido a que, trabaja bajo supuestos. Se concluye que en los emisores de acciones analizados no incide el beta en la rentabilidad ofertada por las empresas vinculadas a la Bolsa de Valores ecuatoriana.
\end{abstract}

Palabras Clave: Mercado de valores de Ecuador, modelo de valuación de activos de capital (CAPM), riesgo sistemático, rentabilidad

\section{CALCULATION OF THE BETAS OF THE CAPITAL ASSET PRICING MODEL AS AN INDICATOR OF PROFITABILITY OF COMPANIES LINKED TO THE ECUADORIAN STOCK EXCHANGE}

\begin{abstract}
The risk when investing in securities belonging to the capital market not only depends on the results of the issuers, but also on the economic environment. Under this context, the objective of the study was to calculate the betas through the application of the Capital Asset Princing Model (CAPM), to know the profitable influence of the companies linked to the Ecuador Stock Exchange. The sample consisted of 35 companies holding shares during the 2014-2019 period. The research was developed under a quantitative approach, through a correlational scope between the variables systematic risk and profitability. The results showed that using systematic risk is not considered as a valuation model for strong financial assets, because it works under assumptions. It is concluded that in the issuers of shares analyzed, the beta does not affect the profitability offered by the companies linked to the Ecuadorian Stock Exchange.
\end{abstract}

Keywords: Ecuador's stock market, capital asset pricing model (CAPM), systematic risk, profitability 


\section{I.INTRODUCCIÓN}

En la actualidad, el poco desarrollo del mercado de valores ecuatoriano hace que las funciones operativas sean ineficientes, debido a que no se cotizan las negociaciones bursátiles dentro de una misma Bolsa de Valores. Existen intereses individuales por parte de la Bolsa de Valores de Guayaquil y Quito, cada una con autorregulaciones, precios y comisiones diferentes pues operan en forma segmentada [1] [2]. En efecto se genera escasa difusión de información de los índices de rentabilidad. Mientras la medición de los riesgos a través de las betas es utilizada con menor frecuencia por los inversionistas. En tal sentido, el mercado de valores no muestra eficiencia, porque los mercados son eficientes si se conoce toda la información de los instrumentos financieros reflejando la relación entre el rendimiento y el riesgo [3]. La ineficiencia en el mercado de valores ecuatoriano se convierte en un factor crucial dentro del país, pues ocasiona desconfianza al tomar decisiones de inversión, el comportamiento humano busca orientación y respuestas que le permitan disminuir la incertidumbre y obtener ventajas al momento de invertir [4].

A nivel mundial cada día son más las empresas y personas que buscan generar ingresos representativos a la inversión realizada. Una forma para lograr este objetivo es la participación en el mercado de valores, al considerar que las tasas de interés que manejan crean ofertas tentadoras y prometen una rentabilidad mayor a la ofertada por el mercado bancario, además existe la seguridad de que las empresas cotizantes cumplirán con sus obligaciones, debido a que para poder pertenecer al mercado de valores deben evaluarse las situaciones económicas y financieras por una calificadora de riesgos, la cual garantiza transparencia. Sin embargo, para muchos sigue siendo un gran dilema decidir si invertir o no, dado que el miedo a lo desconocido se encuentra latente y la estabilidad en el mercado a nivel mundial es incierta, y una crisis global puede desmoronar la estabilidad del mercado de valores.

Por consiguiente, resulta vital destacar que los países que han derivado sus pensamiento y barreras a la inversión presentan un desarrollo superior a los demás gracias a las negociaciones dentro del mercado bursátil, por lo que su economía ha formado el engranaje perfecto hacia el progreso. La interacción de los emisores e inversionistas cada vez adquiere mayor importancia a la hora de potenciar una economía sostenible.

Es quizá por ello que América Latina presenta un desarrollo inferior en el mercado de valores debido a que la relación emisor-inversionista sigue siendo débil, lo cual se refleja de cierta manera en los problemas políticos, económicos y sociales. No se ha logrado alcanzar la participación necesaria para apuntalar a nivel mundial como región. Ecuador, no se queda fuera de esta realidad pues la mayor parte de su población considera que invertir en el mercado de valores es un tema inexplorado, lo que conlleva a que los ecuatorianos asocien la inversión con el fraude o el riesgo a perderlo todo. A pesar de existir información sobre las Bolsas de Valores la difusión de dicha información es asimétrica y no cumple con las expectativas del inversor, de conocer todos los aspectos relevantes de su inversión, quedando de esta forma limitada la canalización de recursos para el financiamiento de diversas actividades económicas y productivas, convirtiéndose como primera opción de ahorro e inversión, el mercado bancario.

La aplicación del CAPM permite establecer la relación entre el riesgo asumido y la rentabilidad esperada a través del riesgo sistemático, que al ser correctamente evaluado se espera que sea directamente proporcional, es decir, a mayor riesgo, mayor rentabilidad. El método de valuación de activos financieros muestra que, para un activo riesgoso en un mercado eficiente, la tasa de retorno es una función de su covarianza. Es decir, explica el comportamiento de una variable en función de lo que hace la otra variable [5].

Es por ello que resulta necesario hacer un estudio de las empresas vinculadas a la Bolsa de Valores del Ecuador, por lo que objetivo del presente trabajo es analizar la influencia de las betas mediante la aplicación del Capital Asset Pricing Model (CAPM) en la rentabilidad de las inversiones realizadas durante el periodo comprendido entre 2014 y 2019.

Este trabajo se encuentra estructurado en 5 secciones: I. Introducción, engloba la situación actual del mercado de valores ecuatoriano y la desconfianza generada por el riesgo al momento de invertir en las distintas Bolsas de Valores. II. Desarrollo, en este apartado se realiza una revisión literaria que permite profundizar criterios sobre el CAPM, la volatilidad, el riesgo sistemático, beta y la rentabilidad. III. Metodología, contiene la justificación de la elección de la muestra y el procedimiento para el cálculo del CAPM. IV. Resultados, se analizan los resultados obtenidos de la aplicación del modelo de valuación de activos financieros reflejados en la tabla CAPM de los emisores de la Bolsa de Valores de Guayaquil y Quito, así como también de los gráficos de dispersión. V. Conclusiones, se deducen las principales conclusiones obtenidas a partir del estudio de riesgo-rentabilidad de las empresas cotizantes. 


\section{II.DESARROLLO}

\section{A.El modelo de fijación de precios de activos de ca- pital (Capital asset pricing model)}

El Capital Asset Pricing Model (CAPM) se basa en la relación entre el riesgo de un activo y su rendimiento [6]. El modelo surge a partir de la teoría moderna del portafolio o teoría moderna de la selección de cartera. A su vez, explica cómo lograr el máximo rendimiento y minimizar el riesgo [7]. La teoría de portafolio fue un paso importante hacia el desarrollo del modelo de valoración de activos financieros CAPM, cuyo objetivo es cuantificar e interpretar la relación entre el riesgo y el rendimiento. A través de esta relación, se puede obtener un punto óptimo en la línea de mercado de valores [8].

El CAPM se calcula a través de beta, que mide el riesgo de mercado que engloba un conjunto de aspectos macroeconómicos que no se pueden diversificar [5]:

1-Los inversionistas son adversos al riesgo, es decir, siempre van a preferir evitar la incertidumbre en sus inversiones financieras.

2-Los inversionistas son tomadores de precios, su poder adquisitivo en el mercado de valores no es lo suficientemente alto como para afectar el precio de los activos financieros. Además, cuentan con idénticas estimaciones subjetivas de las medias, varianzas y covarianzas.

3-Existe un activo de cero riesgos porque promete una rentabilidad certera, conocida con anterioridad.

4-Todos los activos son comerciales, líquidos y divisibles.

5-La tasa de endeudamiento iguala a la tasa de préstamo, la información está disponible para todos los inversores y el riesgo de los activos es similar.

6-El mercado no presenta imperfecciones, costos de transacción ni impuestos.

Este modelo permite el análisis de la rentabilidad financiera, la cual, se obtiene al momento de realizar la inversión de capital en una empresa. Dicha rentabilidad sirve para producir utilidades incluyendo las que no son distribuidas. Se toma en cuenta generalmente un periodo de tiempo y se visualiza como efecto multiplicativo de la rentabilidad económica y el apalancamiento [9]. La rentabilidad no es lo mismo que rendimiento, sino más bien, este es un indicador que permite conocer como varia durante el tiempo la inversión dentro del mercado bursátil, y cuál, ha sido la proporción de los resultados obtenidos con relación al valor del activo.

Al momento de invertir, una gran parte de demandantes de valores únicamente tienen en cuenta, si la empresa es rentable mediante su evaluación de permanecía de actividades, es decir, la expectativa de permanencia de una empresa en el mercado por un determinado periodo. A la vez, descuidan la volatilidad del mercado, ya que, influye en el crecimiento o decrecimiento de la valoración de activos [10]. La desventaja mencionada muestra sensibilidad y puede cambiar al instante por algún factor que no fue considerado al momento de realizar la inversión. Esto afecta de forma directa a los valores financieros.

El nivel de volatilidad señala que, mientras más alto sea, afecta la estabilidad social, ya que produce un incremento en el índice de desempleo, pues, la empresa debilita el factor de producción y se convierte en impredecible el futuro empresarial debido a su alto grado de incertidumbre. Por tal razón, los inversionistas deben asumir todos los riesgos al momento de involucrarse en el mercado de valores, puesto que, no se encuentran exentos de que su inversión obtenga un cambio que desfavorezca su rendimiento.

La incertidumbre se encuentra ligada al riesgo y esta relación es inevitable. Por su parte, la incertidumbre es el desconocimiento del futuro, lo que ocasiona desconfianza. Por otro lado, el riesgo es la probabilidad de tener un resultado no deseado o una pérdida [11]. El riesgo no se puede eliminar, pero al tomar decisiones con estrategias claras basadas en el análisis de datos, puede ser mitigado, lo que permite evitar impactos perjudiciales.

Los inversionistas para impedir que su capital se encuentre expuesto a efectos ocasionados por diversos riesgos que diversifiquen sus inversiones, evitan colocar todo su dinero en un solo activo financiero. Esta diversificación toma en cuenta solo el riesgo no sistemático, y dejan de lado al riesgo sistemático, que se ha convertido en una herramienta imprescindible para la toma de decisiones financieras para aquellos que participan del mercado de valores.

La naturaleza del riesgo sistemático o riesgo de mercado, no genera afectaciones a un sector en particular, sino al mercado en su totalidad. Un ejemplo de este tipo de riesgo, es el momento cuando se produce una crisis financiera, esta impacta directamente a todos los activos de manera simultánea, pues genera un efecto domino. De tal forma, la volatilidad juega un papel crucial y se relaciona con el riesgo sistemático cambiando lo que aparentemente se encontraba predicho. También, se lo conoce como riesgo no diversificable, pues cualquier cambio en el mercado hace que el precio de las acciones fluctué, perturbando a las inversiones incluso si se encuentran colocadas eficientemente.

El inversionista asume el riesgo al adquirir un instrumento financiero que cotice en la bolsa de valores. Esta se relaciona directamente con la empresa emiso- 
ra, la cual se ve afectada por situaciones extrapolares, perdiendo la disponibilidad necesaria para hacer frente a sus obligaciones. En este sentido, el inversor espera que, al aceptar el riesgo sea positivo el retorno del capital invertido.

Para el CAPM, el riesgo sistemático está representado por la tasa de rentabilidad del portafolio de mercado que mide el beneficio generado por el capital invertido y la forma para medirlo es por medio de la beta [5].

Beta $(\beta)$, es una medida del riesgo sistemático relativa a la volatilidad del mercado y la rentabilidad de la acción [12]. Puede proyectar distintos resultados, mayores o menores a 1 [13]:

-Mayor a 1 (valor agresivo), mayor riesgo sistemático, pero resulta favorable cuando el mercado está en crecimiento y desfavorable cuando está en recesión.

-Igual a 1 (valor neutro), igual riesgo sistemático, la variación del mercado y del activo son proporcionales.

-Menor a 1 (valor defensivo), menor riesgo sistemático, la variación del mercado provoca menor variación el activo.

En resumen, mientras más alto sea la beta, mayor será el riesgo. Debido a que, tiene variaciones proporcionales directas del mercado. En ciertas ocasiones, pueden presentarse betas negativas que ocasionan una correlación inversa en el mercado.

Existen diferentes métodos para el cálculo del coeficiente beta del modelo de CAMP [14]:

-El primer método, consiste en la utilización de la covarianza entre la rentabilidad del mercado y de la acción; sobre la varianza de la rentabilidad del mercado. Implica el cálculo de: las estadísticas de regresión, el coeficiente de correlación múltiple, el coeficiente de determinación $\mathrm{R}^{2}$ y el análisis de $\mathrm{R}^{2}$ ajustado como lo demuestra la ecuación 1.

$$
\beta=\frac{\operatorname{COVARIANZA}\left(r_{i}, r_{m}\right)}{\operatorname{VARIANZA}\left(r_{m}\right)}
$$

-El segundo método, consiste en realizar una regresión con los ingresos y las ganancias de las compañías que tienen menor varianza que le precio de sus acciones. Este método presenta limitaciones que se acentúan en mercados emergentes.

-El tercer método, es denominado Bottom-Up, excluye a los datos de los precios pasados, no requiere que la acción cotice en el mercado bursátil, hay que desapalancar las betas y realizar un promedio de las mismas [15].

\section{III.METODOLOGÍA}

La metodología aplicada es de enfoque cuantitativo, porque reúne datos numéricos que permiten el cálculo de las betas y su comprobación por medio de una hipótesis. El alcance aplicado fue correlacional, debido a que, se determinó la vinculación entre el riesgo sistemático (x) y la rentabilidad (y), ofreciendo predicciones de acuerdo a los resultados obtenidos y al comportamiento de las dos variables [16] [17].

\section{A.Recolección de la información y muestra}

Se utilizaron datos históricos de mercado para el cálculo del coeficiente beta, a partir de los precios de las acciones, con un intervalo de 6 años comprendido desde el año 2014 hasta el 2019. Los datos utilizados corresponden a las empresas cotizantes vigentes registradas en el catastro público del mercado de valores de Guayaquil y Quito, pertenecientes al sector financiero y no financiero que fueron inscritas durante el periodo comprendido entre el 03 de enero de 1994 hasta el 31 de diciembre del 2019.

Además, se relacionó a los emisores con el Ranking de participación de las compañías e instituciones en el mercado de valores comprendido desde el año 2002 al 2018, emitido por la Superintendencia de Compañías, Valores y Seguros. Para establecer una muestra relevante para este trabajo que permita cumplir con los objetivos pautados, se trabajó con una muestra de 35 emisores de acciones.

El procedimiento para la determinación del CAPM, fue empleado mediante el primer método que considera la matriz de precios para la ecuación (2)

$$
C A M P=R_{f}+\beta\left(R_{m}-R_{f}\right)
$$

Donde:

CAPM: Rentabilidad esperada

$\mathrm{R}_{\mathrm{f}}$ Rendimiento de un activo libre de riesgo.

$\beta$ : Coeficiente Beta

$\mathrm{R}_{\mathrm{m}}$ : Rendimiento del mercado.

El CAPM requiere para su cálculo el rendimiento del mercado, por lo que se efectuó el cálculo del rendimiento anual de los índices bursátiles de Quito y Guayaquil, con el fin de identificar variaciones relevantes. Debido a que, no está unificado el mercado de valores ecuatoriano se trabajó con dos índices, el IPECU-BVG y el ECUINDEX GLOBAL.

En la figura 1 se observó que en los periodos 20152016, evidenciaron índices negativos. Sin embargo, los periodos 2017-2018 se mostraron en crecimiento. Se lo asocia al cambio de políticas gubernamentales como 
factores externos de afectación del mercado. Con rela-

con relación a los periodos 2017-2018.

ción al año 2019 se presenció un total decrecimiento

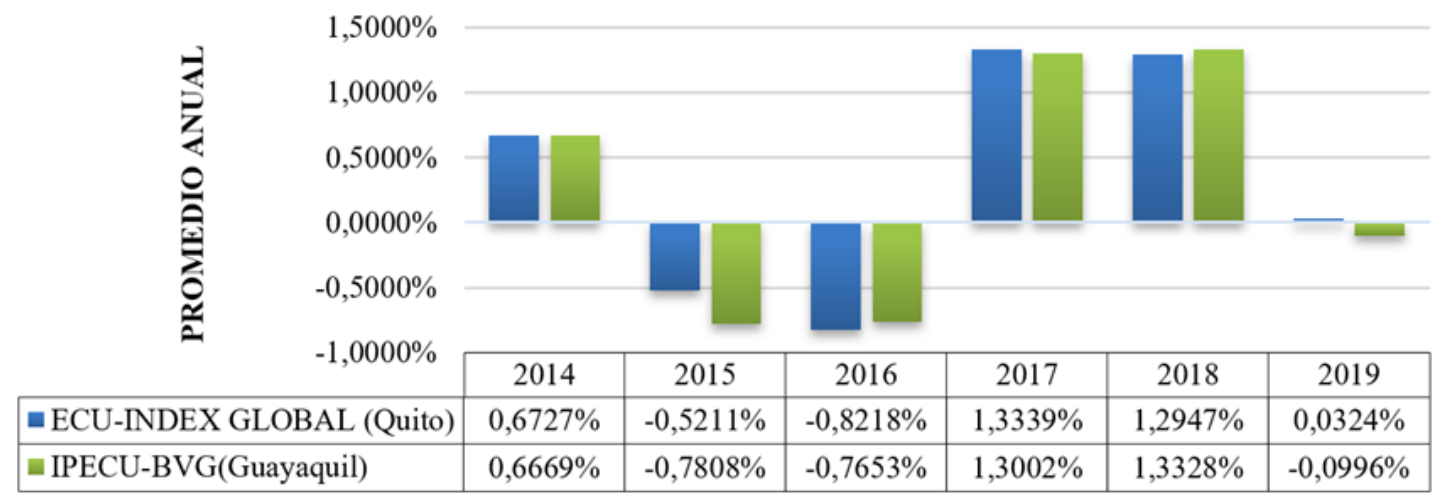

Fig. 1. Rendimiento anual de los índices bursátiles

Fuente: Índices bursátiles del Banco Central del Ecuador

Posterior a ello se efectuó el calculó del promedio de los rendimientos mensuales de los índices bursátiles desde 2014 al 2019, tanto para las empresas cotizantes el cual se requiere para aplicar la fórmula 2 , ya que re- presenta $\mathrm{R}$ _m. La tabla I mostró que el índice bursátil aplicado en Quito genera mejores rendimientos, pues tiene una diferencia de rendimiento en $0,05 \%$.

TABLA I. Rendimiento promedio del mercado y varianza

\begin{tabular}{ccr}
\hline ÍNDICES BURS ÁTILES & RM & \multicolumn{1}{c}{ VARIANZA } \\
\hline IPECU-BVG (Guayaquil) & $0,28 \%$ & 0,0005216 \\
ECU-INDEX GLOBAL (Quito) & $0,33 \%$ & 0,0004224 \\
\hline
\end{tabular}

Fuente: Índices bursátiles del Banco Central del Ecuador

Se ejecutó el procesamiento de datos del rendimiento mensual de los activos, mismos que se encuentran representados por $\mathrm{R}_{-} \mathrm{f}$, de la misma forma se calculó la varianza y covarianza de empresa emisora de accio- nes para tener la base para el cálculo de beta expresado por la fórmula 1, y se llevó a cabo la aplicación de la ecuación (2)perteneciente al modelo de valoración de activos financieros, como se muestran en la tabla II :

TABLA II. CAPM de los emisores cotizantes en la Bolsa de Valores de Guayaquil y Quito

\begin{tabular}{lccccc}
\hline \multicolumn{7}{c}{ BOLSA DE VALORES DE GUAYAQ UIL } & & \\
\multicolumn{1}{c}{ EMISOR } & RF & VARIANZ & COVARIAN & \multirow{2}{*}{ BETA } & CAPM \\
\hline Alicosta Bk Holding S.A. & $-0,23 \%$ & 0,000438238 & $-0,0000574$ & $-0,10998076$ & $-0,0028404$ \\
Banco Bolivariano C.A. & $0,21 \%$ & 0,009646746 & 0,0003649 & 0,699618225 & 0,00255931 \\
Banco Guayaquil S.A & $0,84 \%$ & 0,007465290 & 0,0005124 & 0,982249662 & 0,00285631 \\
Cerro Alto Forestal (Highforest) S.A. & $0,22 \%$ & 0,000211030 & 0,0000081 & 0,015439355 & 0,00225192 \\
Cerro Verde Forestal S.A.(Bigforest) & $0,16 \%$ & 0,000081747 & 0,0000107 & 0,020444641 & 0,0015982 \\
Cervecería Nacional Cn S.A. & $1,17 \%$ & 0,002385583 & 0,0003008 & 0,576581891 & 0,00655471 \\
Continental Tire Andina S. A. & $0,24 \%$ & 0,005207209 & 0,0000162 & 0,031030653 & 0,00243284 \\
Corporación Multibg S.A. & $1,77 \%$ & 0,017604075 & 0,0002371 & 0,454590665 & 0,01090042 \\
Cristalería del Ecuador S.A. Cridesa & $0,06 \%$ & 0,002271210 & 0,0000480 & 0,092065363 & 0,00079608 \\
El Refugio Forestal (Homeforest) S.A. & $0,19 \%$ & 0,000230896 & $-0,0000711$ & $-0,13637635$ & 0,00183356 \\
El Sendero Forestal (Pathforest) S.A. & $0,25 \%$ & 0,000192596 & $-0,0000370$ & $-0,07086329$ & 0,00243833 \\
El Tecal C.A. Elteca & $0,10 \%$ & 0,000036344 & $-0,0000174$ & $-0,03329522$ & 0,00094536
\end{tabular}




\begin{tabular}{|c|c|c|c|c|c|}
\hline Holcim EcuadorS.A. & $-0,27 \%$ & 0,000968258 & 0,0002089 & 0,400452146 & $-0,0005021$ \\
\hline Holding Tonicorp S.A. & $-0,26 \%$ & 0,001399815 & $-0,0000217$ & $-0,04158769$ & $-0,0028162$ \\
\hline Inversancarlos S.A. & $0,14 \%$ & 0,001968309 & 0,0000415 & 0,079648258 & 0,00154273 \\
\hline La Campiña Forestal (Strongforest)S.A. & $0,23 \%$ & 0,000352057 & 0,0000501 & 0,096081722 & 0,00235338 \\
\hline La Colina Forestal (Hillforest) S.A. & $0,23 \%$ & 0,000492642 & 0,0000880 & 0,168690242 & 0,00239519 \\
\hline La Cumbre Forestal (Peakforest) S.A. & $0,27 \%$ & 0,000161926 & $-0,0000100$ & $-0,01917601$ & 0,00270904 \\
\hline La Sabana Forestal (Phinforest) S.A. & $0,35 \%$ & 0,002561769 & $-0,0000855$ & $-0,16391284$ & 0,00357962 \\
\hline La Vanguardia Forestal S.A. & $0,53 \%$ & 0,006962805 & $-0,0002506$ & $-0,48035744$ & 0,00650931 \\
\hline Meriza S.A. & $0,06 \%$ & 0,000041478 & 0,0000188 & 0,03599411 & 0,00071828 \\
\hline Retratorec S.A. & $-0,04 \%$ & 0,001324030 & 0,0000445 & 0,085326847 & $-0,0001755$ \\
\hline Rio Congo Forestal C.A. (Conrioca) & $0,11 \%$ & 0,000055752 & $-0,0000194$ & $-0,03727775$ & 0,00104948 \\
\hline San Carlos S.A. & $-0,89 \%$ & 0,003279293 & 0,0001654 & 0,317055864 & $-0,0052047$ \\
\hline Surpapelcorp S.A. & $0,00099 \%$ & 0,0000197 & $-0,000009$ & $-0,017879787$ & $-0,0000393$ \\
\hline \multicolumn{6}{|c|}{ BOLSA DE VALORES DE Q UITO } \\
\hline EMISOR & RF & $\begin{array}{c}\text { VARIANZ } \\
\text { A } \\
\end{array}$ & $\begin{array}{c}\text { COVARIAN } \\
\text { ZA } \\
\end{array}$ & BETA & CAPM \\
\hline Banco de la Producción S.A. Produbanco & $0,62 \%$ & 0,011833151 & 0,0004590 & 1,086677684 & 0,00306453 \\
\hline Banco Pichincha C.A. & $149,14 \%$ & 156,796586509 & 0,0096904 & 22,94251095 & $-32,649572$ \\
\hline Banco Solidario S.A. & $2,19 \%$ & 0,068547791 & 0,0012946 & 3,065008705 & $-0,0351039$ \\
\hline Cepsa S.A. & $-0,28 \%$ & 0,000547840 & 0,0000705 & 0,166859773 & $-0,0017607$ \\
\hline Conjunto Clínico Nacional Conclina C.A. & $-0,68 \%$ & 0,003864391 & 0,0001233 & 0,291909946 & $-0,0038785$ \\
\hline Corporación Favorita C.A. & $-0,45 \%$ & 0,006485508 & 0,0006652 & 1,574891252 & 0,00783922 \\
\hline Hotel Colon Internacional C.A. & $0,46 \%$ & 0,014466057 & 0,0001268 & 0,300297542 & 0,00424375 \\
\hline Industrias Ales C.A. & $-1,33 \%$ & 0,004455035 & $-0,0000016$ & $-0,00367135$ & $-0,0133557$ \\
\hline Mutualista Pichincha & $-0,24 \%$ & 0,001463261 & $-0,0000519$ & $-0,1227915$ & $-0,0030877$ \\
\hline Superdeporte S.A. & $0,43 \%$ & 0,000879314 & $-0,0000098$ & $-0,02310688$ & 0,00433278 \\
\hline
\end{tabular}

Fuente: Analytica Securities C.A. Casa de Valores, Superintendencia de Compañías, Valores y Seguros.

\section{IV.RESULTADOS}

En la TABLA I., se mostraron los rendimientos anuales de los índices bursátiles existentes en el Ecuador, el IPECU-BVG de Guayaquil que refleja el 0,28\% de rendimiento de mercado y el ECUINDEX GLOBAL de Quito que alcanza 0,33\% durante el lapso comprendido desde el año 2014 al año 2019. Así como la variabilidad de los datos mensuales en este periodo. Existe una diferencia entre rendimientos del $0,05 \%$ lo que ofrece mayor rendimiento en la Bolsa de Valores de Quito.

De acuerdo a TABLA II. Se logró apreciar el promedio del rendimiento de los activos de las 35 empresas cotizantes, reflejando que tanto en la Bolsa de Valores de Guayaquil como en la de Quito existen rendimientos negativos lo que se traduce a que las fluctuaciones en el precio de las acciones de dichas empresas tienden a bajar por la gestión de la empresa o a su vez por la ley de oferta y demanda.

Los valores obtenidos de beta por medio de la ecuación (1), muestran que el 11\% de los emisores analizados tienen una beta mayor a 1 lo que significa que la acción cotizada en la bolsa de valores presenta una mayor volatilidad que el mercado ocasionando mayor riesgo sistemático. Mientras que, el 37\% refleja una beta nega- tiva que significa que las acciones subirán o bajaran de manera inversa que el mercado y el $51 \%$ restante presenta un resultado de beta menor a 1 lo que se interpreta como poca volatilidad, es decir el precio de las acciones varía menos que el mercado, por lo que corren un riesgo sistemático menor.

Cabe señalar, que en la figura I se evidenció el rendimiento anual del mercado de las Bolsas de Valores ecuatorianas, presentando resultados similares debido a la mínima variación entre la ciudad de Guayaquil y Quito. El año 2015 y 2016 presentan un rendimiento del mercado negativo, esto se debe a la disminución de depósitos bancarios, pues la banca mantiene un porcentaje de sus pasivos en el mercado bursátil otorgando la liquidez necesaria para los inversionistas institucionales.

Los emisores pertenecientes a la Bolsa de Valores de Quito representan el 29\% del total de la muestra, sucede lo mismo a la hora de analizar todos los emisores vigentes por Bolsa de Valores, siendo el porcentaje de participantes en el mercado de valores mayor en Guayaquil. No obstante, de acuerdo a la tabla podemos comprobar que Quito tiene mayor rendimiento de mercado. 


\section{A.Figuras CAPM}

Las figuras representan los valores más relevantes del cálculo beta dentro del estudio del CAPM, con la línea del mercado de valores se refleja que la rentabilidad esperada de todos los activos debe permanecer sobre la línea de mercado, asegurando que sea proporcional al riesgo sistemático.

El eje horizontal representa la rentabilidad del índice bursátil y el eje vertical la rentabilidad de la acción, la figura 2 y 5 representan una beta negativa por lo que, si las acciones suben el mercado tiende a bajar y viceversa.

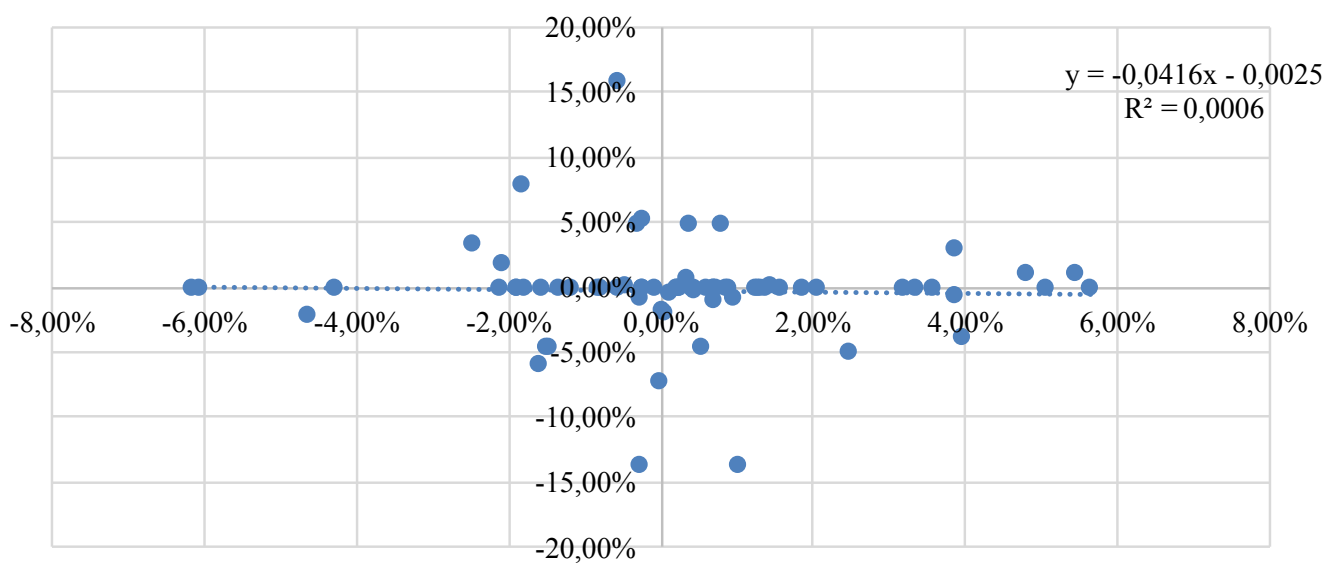

Fig. 2. Dispersión Holding Tonicorp S.A.

La figura 2 de dispersión del Banco Bolivariano C.A., al tener una beta igual a 0,6996 y estar próximo a 1 , muestra como ésta se mueve en la misma dirección que el mercado, es decir sin las variaciones que van a la par. Al ser un valor menor existe menor riesgo sistemático.

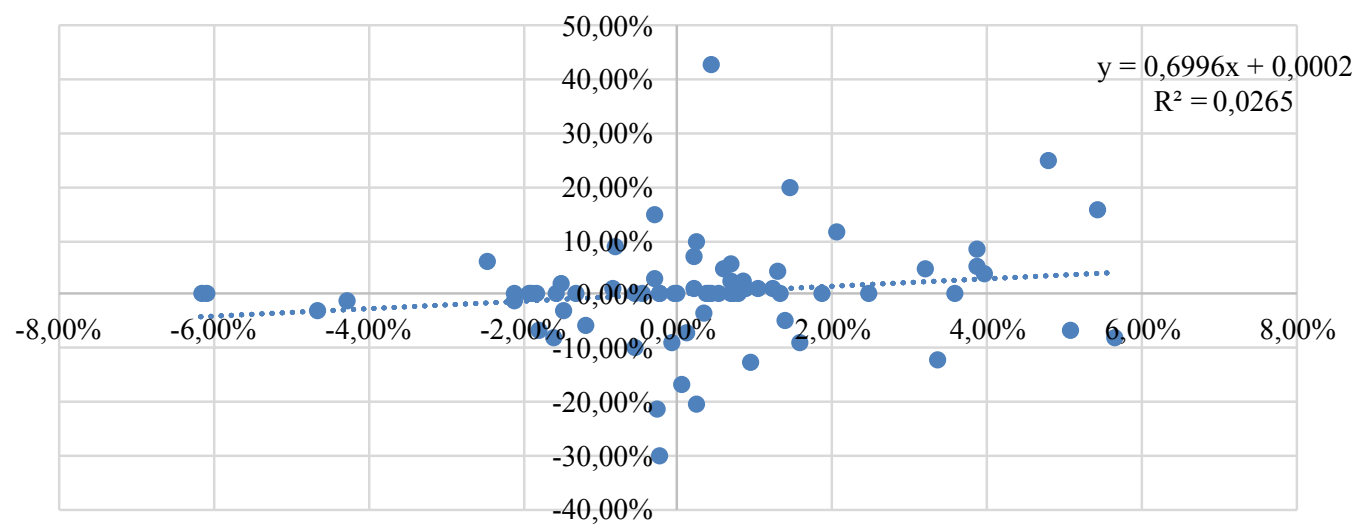

Fig. 3. Dispersión del Banco Bolivariano C.A

La figura 4 muestra la línea de mercado del Banco Pichincha C.A. con una beta de 22,94 la cual indica que el precio de las acciones es más volátil y se corre como inversor mayor riesgo sistemático, además la gráfica tiene un valor pronunciado de alza de precio por lo que se puede ver reflejado en el valor alto de beta. 


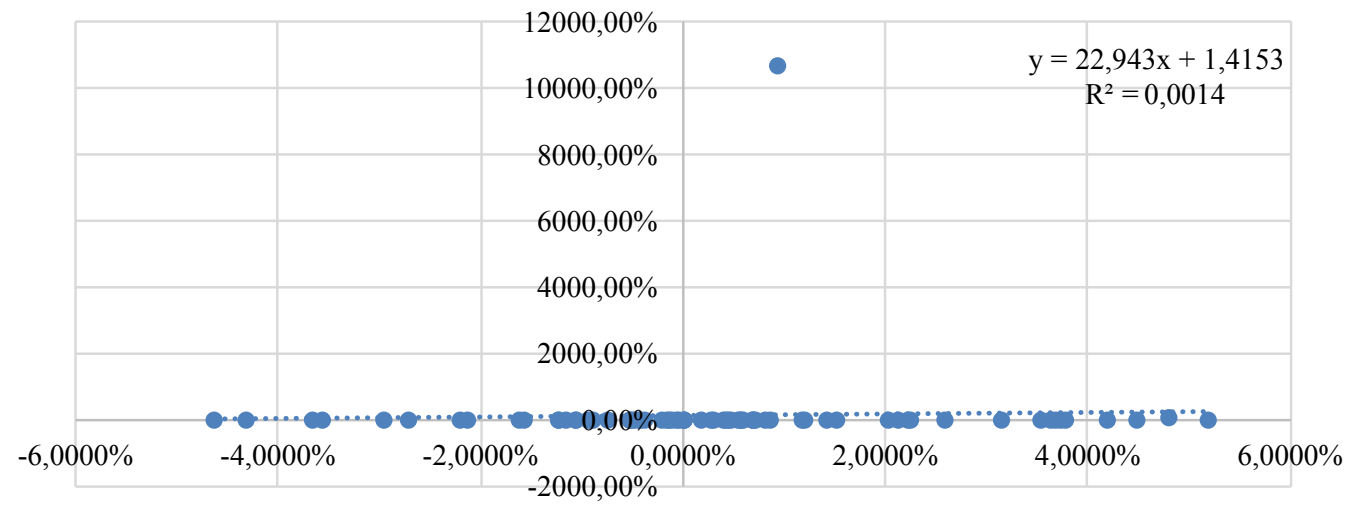

Fig. 4. Dispersión del Banco Pichincha C.A.

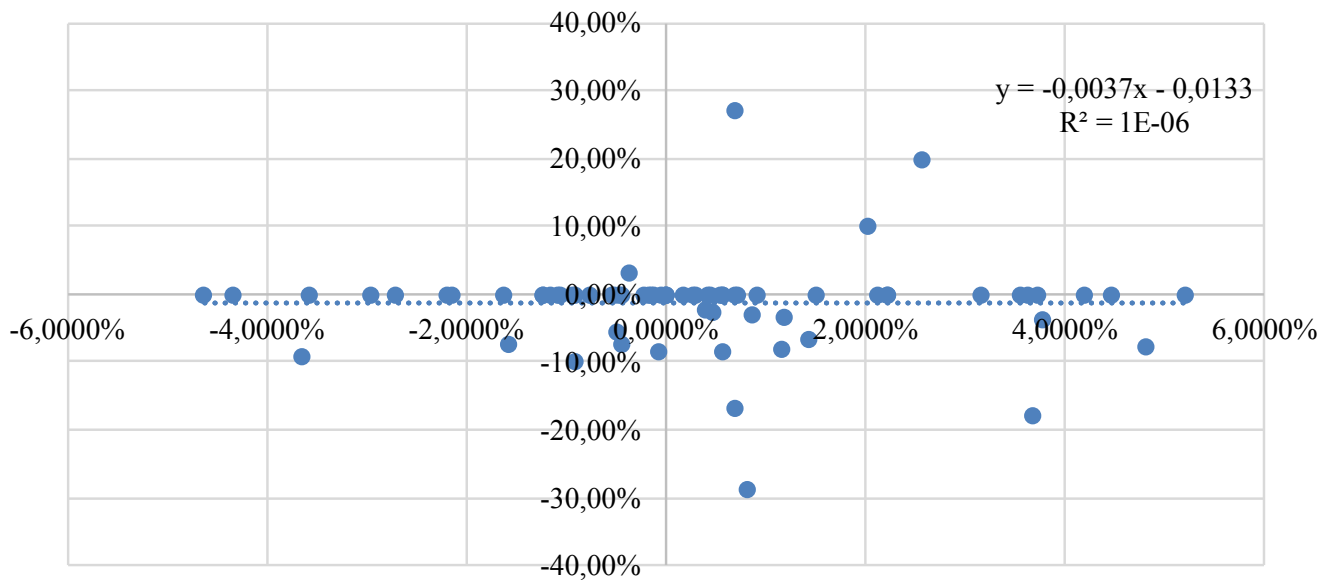

Fig. 5. Dispersión de Industria Ales C.A.

\section{V.CONCLUSIONES}

El mercado de valores ecuatoriano debe trabajar hacia el perfeccionamiento e integración de la Bolsa de Valore de Quito y Guayaquil, puesto que resulta una oportunidad para dinamizar la economía del país. Al ser estas las entidades encargadas de canalizar recursos financieros para diversos sectores de producción por medio de la negociación de valores. Resulta vital atraer nuevos participantes al mercado bursátil y generar estrategias que permitan su permanencia, pues generan beneficios a nivel nacional como el incremento de empleo, gracias al aumento de las operaciones empresariales y personal como la maximización de capital tanto de las empresas cotizantes como de los inversionistas, al obtener un rendimiento sobre su inversión.

El intervalo de tiempo para analizar el CAPM es importante ya que al seleccionar un tiempo corto ocasiona ciertas desventajas debido a que el mercado de valores es muy volátil, tanto el riesgo sistemático como no sistemático influyen en las cotizaciones y puede verse reflejado en el beta.

Una limitante del CAPM es la determinación minuciosa de la beta, debido a la sensibilidad de este valor en los procesos de cálculo. Una variación brusca de la beta indica posibles causas de cambios significativos en esta medida, pese a que su formulación es sencilla al varia por diversos factores macroeconómicos de modo relevante la estimación a base de datos históricos quedaría obsoleta para estimaciones futuras por su inestabilidad. No es seguro que los resultados obtenidos se mantengan durante un periodo prolongado de la misma forma que los 6 años analizados.

El modelo CAPM puede presentar debilidades en las predicciones, ya que solo considera el riesgo sistemático, sin embargo, la determinación de la beta ayuda en la comprensión de la rentabilidad ante movimientos del mercado.

El cálculo de la beta no es un factor excluyente de la rentabilidad de las empresas vinculadas a la Bolsa de Valores ecuatoriana, lo que resulta significativo para los 
inversionistas, quienes pueden obtener la rentabilidad de su inversión pese a los valores agresivos de la beta.

\section{REFERENCIAS}

[1]F. J. Riofrio, “Análisis comparativo del mercado de valores: Ecuador, Colombia, Perú, y propuesta de medidas para el desarrollo del caso ecuatoriano.," Universidad Internacional del Ecuador, 2019.

[2]C. Albuja, "Integración del Mercado de Valores en Ecuador, factibilidad del proyecto," Pontificia Universidad Católica del Ecuador, 2013.

[3]E. Quiroga, "Eficiencia en los mercados financieros y predicción de precios de los activos," Ciencias Adm., vol. 10, p. 11, 2017, [Online]. Available: http://www. redalyc.org/articulo.oa?id=511653854005.

[4]J. B. Duarte Duarte, L. H. Talero Sarmiento, and K. J. Sierra Suárez, "Evaluación del efecto de la psicología del inversionista en un mercado bursátil artificial mediante su grado de eficiencia," Contaduria y Adm., vol. 62, no. 4, pp. 1345-1360, 2017, doi: $10.1016 / \mathrm{j}$. cya.2017.06.007.

[5]F. Rubio, "Capital Asset Pricing Model (Capm) Y Arbitrage Pricing Theory (Apt): Una Nota Técnica," Recuper., January 2004, p. 4, 2004, doi: http://econwpa. repec. org/eps/fin/papers/0402/0402007. pdf.

[6]W. Sharpe, "Capital Asset Prices A Theory of Market Equilibrium under Conditions of Risk-convertido ES," Wiley-Blackwell, 1964.

[7]O. Mejía, "Discusión sobre la teoría moderna del portafolio. Aplicación de la internacionalización del portafolio, incluyendo el caso colombiano," Estud. Gerenciales, pp. 103-116, 2004.

[8]E. Sansores, "El modelo de valuación de activos de capital aplicado a mercados fi nancieros emergentes," Contaduria y Adm., no. 226, pp. 93-111, 2008.

[9]A. Sanchez, "La Rentabilidad Economica Y Financiera de la Gran Empresa Española," Rev. Española Financ. y Contab., vol. XXIV N 78, p. 159 179, 1994, [Online]. Available: https://www.mendeley.com/ viewer/?fileId=4725a92a-52e8-75a5-78c5-00e347 6e79ab\&documentId=414ff01 c-9b08-3eba-a6a4-beb$38 \mathrm{dd} 799 \mathrm{f} 7$.

[10]F. Garcia, J. Gonzalez, G. Rueda, and J. Oliver, "Characterization of capital markets of Latin America, 2000-2016: A comparative analysis," Espacios, vol. 39, no. 50, p. 9, 2018.

[11]B. Tocabens, "Definiciones acerca del riesgo y sus implicaciones," Rev. Cubana Hig. Epidemiol., vol. 49, no. 3, pp. 470-481, 2011.

[12]C. Martínez, J. Ledesma, and A. Russo, "Particularidades del Modelo de Fijción de Precios de Activos de Capital (CAPM) en Mercados Emergentes," 2013.
[Online]. Available: www.unq.edu.ar.

[13]A. Támara, I. Chica, and A. Montiel, "Metodología de cálculo del beta: Beta de los activos, beta apalancado y beta corregido por cash," Espacios, vol. 38, no. 34, 2017, [Online]. Available: https://www.revistaespacios. com/a17v38n34/a17v38n34p15.pdf.

[14]C. Martínez, J. Ledesma, and A. Russo, "Calculating beta models to apply in Capital Asset Pricing Model: The case of Argentina," Estud. Gerenciales, vol. 30, no. 131, pp. 200-208, 2014, doi: 10.1016/j.estger.2014.03.002.

[15]I. Conte, "La inestabilidad de la $\beta$ como medidor del riesgo sistemático y sus implicaciones en el modelo de valoración CAPM.," Universidad Pontificia Comillas, 2014.

[16]R. Hérnandez, C. Férnandez, and M. Baptista, Mewtodología de la investigación, Mc Graw Hi. México D.F., 2010.

[17]E. Cabezas, D. Andrade, and J. Torres, Introducción a la Metdología de la Investigación Científica, David Andr., vol. 66. 2018.

[18]Banco Central del Ecuador, "Índices Bursátiles", Available: https://www.bce.fin.ec/index.php/component/k2/item/149-indices-burs\%C3\%A1tiles, 2019

\section{RESUMEN CURRICULAR}
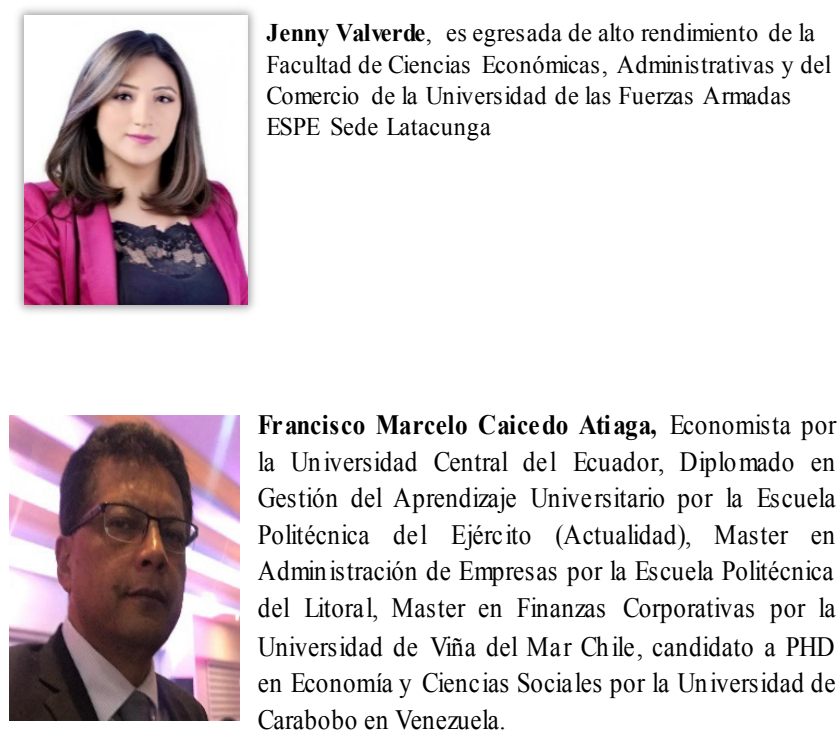

Francisco Marcelo Caicedo Atiaga, Economista por la Universidad Central del Ecuador, Diplomado en Gestión del Aprendizaje Universitario por la Escuela Politécnica del Ejército (Actualidad), Master en Admin istración de Empresas por la Escuela Politécnica del Litoral, Master en Finanzas Corporativas por la Universidad de Viña del Mar Chile, candidato a PHD en Economía y Ciencias Sociales por la Universidad de Carabobo en Venezuela. 